\title{
Dystrophie unguéale médiane
}

\author{
Morteza Khodaee MD MSP, Naomi Kelley BS, Sabrina Newman MD
}

Citation : CMAJ 2020 December 14;192:E1810. doi : 10.1503/cmaj.201002-f

Voir la version anglaise de l'article ici : www.cmaj.ca/lookup/doi/10.1503/cmaj.201002

$\mathbf{U}$ n homme de 34 ans a consulté son médecin de famille au sujet de lésions indolores bilatérales à l'ongle du pouce perdurant depuis 20 ans. Le patient n'avait aucun antécédent de trauma ou d'infection à l'ongle. Les ongles des 2 pouces présentaient une dépression linéaire centrale assortie de crêtes transversales parallèles, donnant un aspect en branches de sapin (figure 1). Il n'y avait aucun érythème ni chaleur autour des ongles, qui n'étaient pas douloureux au toucher. Ses autres ongles doigts et orteils - n'étaient pas affectés. Nous avons donc posé un diagnostic de dystrophie unguéale médiane.

La dystrophie unguéale médiane, qu'on désigne aussi sous le nom de dystrophia unguis mediana canaliformis, dystrophie médiane canaliforme ou solénonychie, est une affection de l'ongle peu courante qui touche les hommes et les femmes en proportions égales. Possiblement associée à un microtraumatisme de la matrice unguéale, l'affection est souvent symétrique et touche le plus souvent les pouces ${ }^{1-3}$. L'onychotillomanie, les tumeurs des ongles, le kyste mucoïde du doigt et la trachyonychie (ongles rugueux) sont d'autres diagnostics courants se manifestant par des défauts longitudinaux de l'ongle $\mathrm{e}^{4-6}$. La recherche des antécédents détaillés devrait se concentrer sur les accidents de travail et les habitudes. Parmi les autres affections à envisager, mentionnons l'onychomycose, le lichen plan, la maladie de Darier et le psoriasis ${ }^{3-5}$. Pour écarter le diagnostic d'onychomycose, il est suggéré d'utiliser une préparation d'hydroxyde de potassium, une technique de fluorescence ou une culture fongique. Les signes d'une infection fongique incluent la chromonychie (coloration fongique blanchâtre ou jaunâtre), l'onychauxis (épaississement de l'ongle) et l'onycholyse avec débris sous-unguéaux ${ }^{4,6}$. En l'absence d'autres lésions de la peau ou des muqueuses (p. ex., stries de Wickham), le diagnostic de lichen plan est très peu probable ${ }^{3,5}$. Les fissures des ongles dues au lichen plan ont tendance à être irrégulières et ne sont généralement pas associées à des crêtes ou à un agrandissement des lunules ${ }^{3,5}$.

Bien que la dystrophie unguéale médiane puisse être traitée par l'application topique de corticostéroïdes, de tacrolimus et de

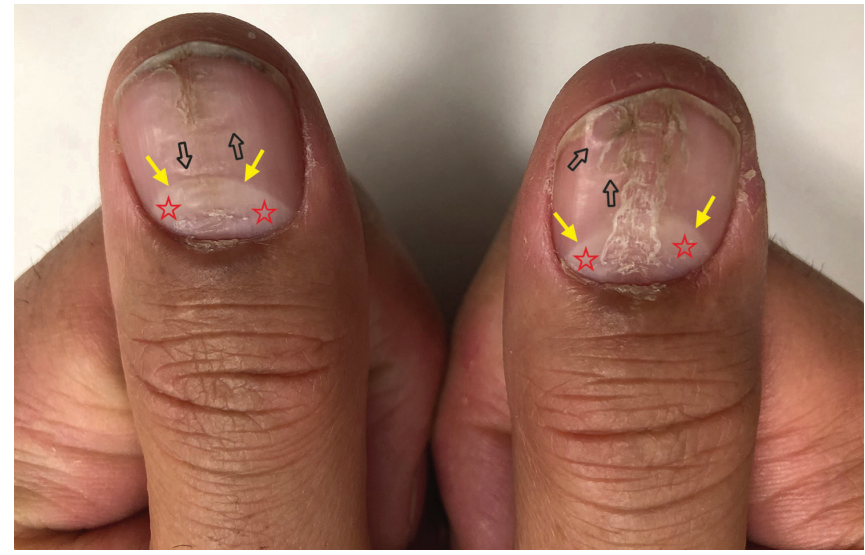

Figure 1 : Dystrophie unguéale médiane chez un homme de 34 ans. Ongles du pouce présentant une fissure lamellaire longitudinale assortie de crêtes transversales parallèles donnant un aspect de plume (flèches vides), un agrandissement des lunules (zones blanches [étoiles rouges] entre le sillon latéral de l'ongle et le reste du limbe [flèches jaunes]) et une hyperpigmentation et une hyperkératose focales associées au sillon proximal.

tazarotène, il n'existe pas de preuves claires que les changements d'apparence de l'ongle disparaîtront ${ }^{1-3}$. Le patient a choisi de ne suivre aucun traitement, puisqu'il ne s'agissait que d'une question d'apparence.

\section{Références}

1. Kota R, Pilani A, Nair PA. Median nail dystrophy involving the thumb nail. Indian J Dermatol 2016;61:120.

2. Pathania V. Median canaliform dystrophy of Heller occurring on thumb and great toe nails. Med J Armed Forces India 2016;72:178-9.

3. Richert B, Caucanas M, André J. Diagnosis using nail matrix. Dermatol Clin 2015;33:243-55.

4. Tully AS, Trayes KP, Studdiford JS. Evaluation of nail abnormalities. Am Fam Physician 2012;85:779-87.

5. Biesbroeck LK, Fleckman P. Nail disease for the primary care provider. Med Clin North Am 2015;99:1213-26.

6. Wollina U, Nenoff P, Haroske G, et al. The diagnosis and treatment of nail disorders. Dtsch Arztebl Int 2016;113:509-18.
Intérêts concurrents : Aucun déclaré.

Cet article a été révisé par des pairs.

Les auteurs ont obtenu le consentement du patient.

Affiliations : Département de médecine familiale et d'orthopédie (Khodaee), École de médecine de l'Université du Colorado, clinique AFW, Denver, Colo.; École de médecine de l'Université du Colorado (Kelley); Département de dermatologie (Newman), École de médecine de l'Université du Colorado, Aurora, Colo.
Propriété intellectuelle du contenu : Il s'agit d'un article en libre accès distribué conformément aux modalités de la licence Creative Commons Attribution (CC BY-NC-ND 4.0), qui permet l'utilisation, la diffusion et la reproduction dans tout médium à la condition que la publication originale soit adéquatement citée, que l'utilisation se fasse à des fins non commerciales (c.-à-d., recherche ou éducation) et qu'aucune modification ni adaptation n'y soit apportée. Voir : https:// creativecommons.org/licenses/by-nc-nd/4.0/deed.fr.

Correspondance : Morteza.khodaee@cuanschutz.edu 